\title{
Successful Delivery of A Full-Term Pregnant Patient with Acute Stanford A Aortic Dissection: A Case Report
}

\author{
Mountajab Saleh ${ }^{1}$, Alaa Raslan ${ }^{2 *}$, Monzer Khalil ${ }^{3}$ and Akil Khaddam ${ }^{1}$ \\ ${ }^{1}$ Division of Cardiac Surgery, Tishreen University Hospital, Lattakia, Syria \\ ${ }^{2}$ Division of Cardiology, Tishreen University Hospital, Lattakia, Syria \\ ${ }^{3}$ Division of Obstetrics and Gynecology, Soued Hospital, Lattakia, Syria
}

\begin{abstract}
*Corresponding author: Alaa Raslan, Division of Cardiology, Tishreen University Hospital, Lattakia, Syria.
\end{abstract}

Received Date: : November 04, 2019

Published Date: November 12, 2019

\begin{abstract}
Aortic dissection occurring during pregnancy is a rare but potentially lethal condition for both the mother and the fetus. We report a case of Stanford An aortic dissection in a full-term pregnant woman with no risk factors who was admitted for an elective caesarean section. The patient underwent emergent ascending aorta replacement immediately after caesarean section with a favorable maternal and fetal outcome.
\end{abstract}

Keywords: Aortic dissection; Pregnancy; Echocardiography; Caesarean section

\section{Introduction}

Aortic dissection is often associated with catastrophic outcomes that can lead to a significantly higher risk of mortality in pregnant women [1]. Approximately 50\% of aortic dissections in young women are associated with pregnancy $[2,3]$. This condition is often associated with a genetic or anatomic predisposition including Marfan syndrome, or bicuspid aortic valve [4]. The clinical presentation is variable. The most common symptom is chest pain, usually of sudden onset and often described as tearing or "knife-like" pain. It is present in more than $90 \%$ of patients with aortic dissection [2]. Other findings may include hypertension, hypotension and manifestations of end-organ ischemia.

\section{Case Presentation}

A full term pregnant, 38 -year-old woman was admitted to hospital at the planned day for delivery. Her past medical history included a caesarean section three years ago and gestational hypertension during the former pregnancy. The indication for elective caesarian section was the prior caesarean. During the current pregnancy she received appropriate care and follow up, and no hypertension was reported.

The patient complained of severe episodes of diarrhea and vomiting with chest pain a few hours before programmed admission. The obstetrician decided to delay delivery in order to further evaluate the patient and her fluid state. A cardiovascular consultation was requested due to an audible heart murmur on auscultation. Detailed history revealed several episodes of diarrhea and vomiting followed by severe constricting central chest pain. The pain had an abrupt onset five hours before admission and lasted for 15 minutes before becoming less severe. Her pain radiated to the jaw and was increased with inspiration. The patient also reported orthopnea during the last few days. On clinical examination a significant interarm blood pressure differential was found (right arm: 140/80 mmHg, left arm: 100/60 mmHg), heart rate was 75 bpm and Spo 2 was $98 \%$ in room air. No features of Marfanoid habitus was noted, no jugular distention was found, and lungs were clear with no obvious rales. Heart auscultation revealed a diastolic murmur (3/6) heard at the aortic area and at the left sternal border. Mild pitting edema was found on lower extremities examination and peripheral pulses were palpable. ECG exhibited first degree atrioventricular block (unknown to be previously present) with no other significant findings. On transthoracic echocardiography, significant ascending aorta dilation $(50 \mathrm{~mm})$ was noted. The aortic valve was tricuspid with moderate regurgitation (Figure1).

Depending on the high clinical probability of aortic dissection the patient underwent contrast-enhanced CT. CT revealed dilation of the ascending aorta (maximal $54 \mathrm{~mm}$ ) with a double-lumen sign 
(Figure2) and the diagnosis of acute aortic dissection (DeBakey II, Stanford A) was established. The patient underwent emergent caesarean section under general anesthesia in the cardiac operation room. The cardiac surgical team was ready, and the cardiopulmonary bypass was prepared in the operating room before the cesarean section. Immediately after the successful delivery of a viable baby, emergent ascending aorta replacement was performed. Femoral artery cannulation was used to establish cardiopulmonary bypass and using a dacron graft, aortic replacement was done with performing repair of the aortic valve (Figure3).

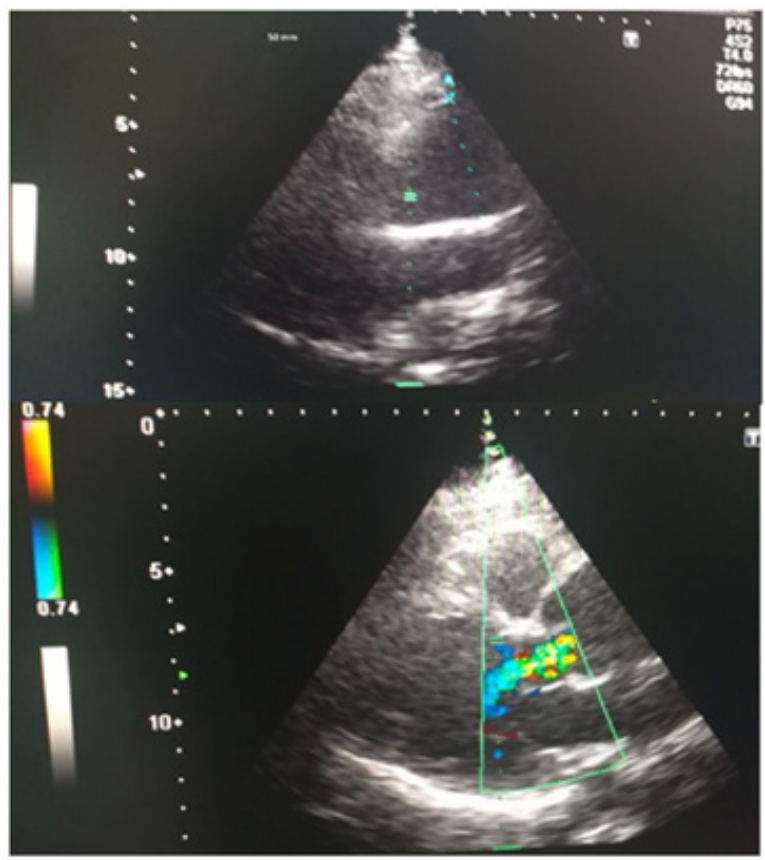

Figure 1: TTE shows aortic dilation with moderate AR.

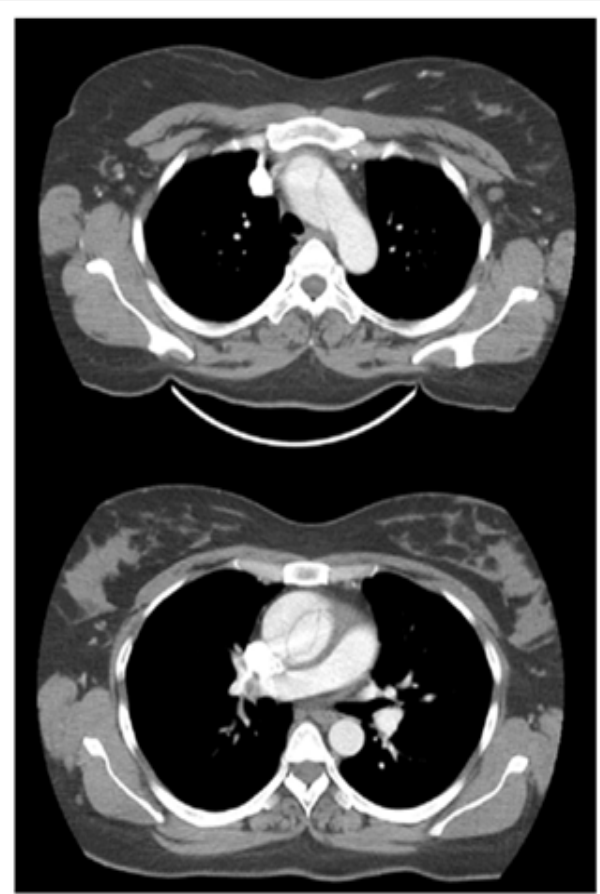

Figure 2: Contrast-enhanced CT shows Double-lumen sign.

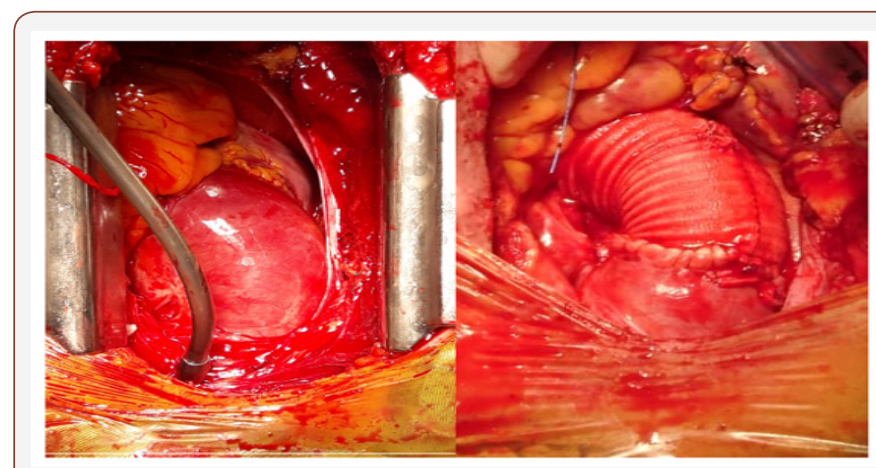

Figure 3: Ascending aorta replacement.

The patient recovered well during hospital stay with no significant complications reported, and after six days in the intensive care unit the patient was discharged in stable condition with her infant baby. TTE on discharge exhibited only mild aortic regurgitation. The patient was advised to perform genetic testing later.

\section{Discussion}

Aortic dissection during pregnancy is a rare but potentially lethal condition for both the pregnant woman and the baby. Eighty percent of the dissections during pregnancy are a type A dissection, and $87.5 \%$ of these cases occur in the pre-partum period [4]. Aortic dissection mostly occurs during the third trimester, which is a period of maximal hemodynamic stress. Both hemodynamic and structural changes occur in the aortic wall during pregnancy. The hemodynamic stresses peak at 32 weeks gestation because of a $50 \%$ increase in intravascular volume. Furthermore, stroke volume and heart rate also increase, resulting in an increased cardiac output of about $40 \%$ [5]. Estrogen has been found to alter lumen diameter and compliance of vascular wall [4].

This combination of vascular stress and change of aortic tunica media architecture in an already abnormal aorta predisposes to dissection. Time to diagnosis is often delayed by a low degree of suspicion or atypical presentation. Sometimes difficulties in investigating pregnant women play a major role. In acute type A aortic dissection, surgery is the treatment of choice. This case has a mortality of $50 \%$ within the first 48 hours if not operated [6]. This critical event represents a unique condition where careful appreciation of potential complications must consider both the patient and her fetus, and that requires special medical efforts and high cooperation of multiple teams. There have been several case reports of successful management of Stanford type A aortic dissection in pregnant women with a cesarean section followed by emergency aortic surgery $[1,4]$. In some previous reports, a sternotomy was performed prior to the cesarean section to facilitate urgent cardiopulmonary bypass (CPB) for possible hemodynamic instability during the cesarean section [4]. However, in our case a cesarean section was performed safely and was followed immediately by aortic surgery with CPB, without a prior sternotomy. Most reported cases are associated with preexisting connective tissue disease such as Marfan's syndrome (50\% of reported cases) and Ehlers-Danlos syndrome, systemic hypertension or congenital 
heart disease such as coarctation and bicuspid aortic valve [1] However, in our case the patient was remarkably different from most of the other patients reported in literature because she had none of these predisposing factors. Clinical presentation was also misleading but careful examination raised the suspicion about aortic dissection.

\section{Conclusion}

Occurrence of type A aortic dissection in pregnant women is rare but potentially catastrophic.

A high index of suspicion and timely investigations are necessary to facilitate fast definitive management even in the absence of previously known risk factors. Appropriate surgical strategies and collaboration of multiple teams are necessary to optimize outcome.

\section{Acknowledgement}

None.

\section{Conflict of Interest}

No conflict of interest.

\section{References}

1. Yuan SM (2013) Aortic dissection during pregnancy: a difficult clinical scenario. Clin Cardiol 36: 576-584.

2. Finkelmeier BA, Marolda D (2001) Aortic dissection. J Cardiovasc Nurs 15: $15-24$.

3. Zeebregts CJ, Schepens MA, Hameeteman TM, Morshuis WJ, de la Riviere AB, et al. (1997) Acute aortic dissection complicating pregnancy. Ann Thorac Surg 64(5): 1345-1348.

4. Ch'ng SL, Cochrane AD, Goldstein J, Smith JA (2013) Stanford type a aortic dissection in pregnancy: a diagnostic and management challenge. Heart Lung Circ 22: 12-18.

5. Hall ME, George EM, Granger JP (2011) The heart during pregnancy. Rev Esp Cardiol 64(11): 1045-1050.

6. Erbel R, Aboyans V, Boileau C, Bossone E, Bartolomeo RD, et al. (2014) ESC guidelines on the diagnosis and treatment of aortic diseases: document covering acute and chronic aortic diseases of the thoracic and abdominal aorta of the adult. The Task Force for the Diagnosis and Treatment of Aortic Diseases of the European Society of Cardiology (ESC). Eur Heart J 35: 2873-2926. 